\title{
A New Model for History Dependent Saturation Functions in Reservoir Simulation
}

\author{
KRIEBERNEGG, Michael, Mining University Leoben \\ HEINEMANN, Zoltán E., Mining University Leoben
}

Paper presented at the 5th European Conference on the Mathematics of Oil Recovery, Leoben, Austria, 3-6 Sept. 1996

\begin{abstract}
A new model for treating dynamic hysteresis in saturation functions for two and three phase reservoir simulation studies has been developed. It is incorporated in a three dimensional, three phase, fully implicite reservoir simulator.

It has been experimentally observed that capillary pressure and relative permeabilities do not only depend on the saturation change itself, they depend on the direction of saturation change, too, which is called as 'hysteresis'. In simulations with changing displacement directions, accounting for hysteresis in saturation functions leads to more realistic predictions of reservoir behavior.

In this model, dynamic values of saturation functions are determined by remembering the history in saturation. Normalized experimental functions are used to predict the virtual values of saturation functions at any saturation value for given displacement conditions. The influence of the maximum historical non-wetting phase saturation on the residual one is accounted for. The basic principles of the model are derived from experimental observations.

The model allows to select those 'level of hysteresis' which is in accordance with the expected displacement mechanisms. Saturation functions of rock regions can be defined by entering the endpoints of curves only and 'scaling' the function of another rock region with known function shape between the endpoints, too.

The hysteresis model was validated by parametric studies of several hypothetical systems representing typical displacement mechanisms in reservoirs.
\end{abstract}

\section{INTRODUCTION}

The purpose of this paper is to report a new model for dynamic treating of hysteresis in saturation functions that allows the modeling of hysteretic flow in multi-cycle drainage-imbibition displacement processes. The paper focuses on the presentation of the applied principles and the mathematical treatment. Some examples are presented to demonstrate the major effects on simulation studies.
The existence of hysteresis in the relationship of capillary pressure and/or relative permeabilities to saturation has been recognized by many authors $[3,5,6,11,12,15]$.

Evrenos [4] found, that multi-cycle drainage-imbibition processes differ from the non-cyclic displacement. He recommends to consider dynamic hysteresis exhibited by phase relative permeability and capillarity for changing directions in saturation change.

In some of the processes to be simulated by present day simulators, one set of capillary pressure functions is used for initialization and/or simulation of part of the reservoir. Additionally, one set of relative permeability curves is used and it is assumed that saturation changes occur in a given direction - drainage or imbibition - for most of the simulation [8]. This simplified treatment of saturation functions will give accurate predictions of reservoir behavior only, if it is sure that mainly the assumed displacement processes (either displacement of a nw phase by a wetting phase and vice versa) occur during the simulation.

Some authors reported that the results of reservoir simulations with and without including the effect of hysteresis are significantly different for simulation of such displacement processes as water-coning with variable rates, gas-cap shrinkage, waterflooding in the presence of frè gas, simulation of cyclic steam processes and simulation of cyclic gas storage processes $[1,3,4,5,6]$. One set of saturation functions will be not sufficient for describing of such displacement processes.

As there is no theoretical analysis available to predict the values of drainage and imbibition curves at any saturation values, empirical methods have to be applied $[2,8,14]$.

Killough [8] developed a capillary pressure model that uses interpolation with a regression parameter to generate intermediate scanning curves. His model allows the input of bounding imbibition and drainage experimental curves, 
which meet at the residual nw phase saturation. Much of the data in the literature do not show this characteristic and the model does not reflect the saturation history of a real reservoir. In the treatment of relative permeability hysteresis, Killough is using parametric interpolation and normalized experimental data for calculating intermediate imbibition curves. He assumes, that imbibition curves are reversible. Experimental data show the non-reversibility of imbibition relative permeabilities $[3,4,6,15]$.

Evrenos [4] presented a numerical simulator for modeling of hysteretic flow in aquifer storage reservoirs. His hysteresis model accounts for multi-cycle drainageimbibition processes during gas storage operations. The model is based on multi-cycle capillarity and relative permeability tests. He considers that a primary drainage process can be stopped at any stage before the residual wetting phase saturation is reached, even it is possible in his model to go through several drainage-imbibition cycles before wetting phase is completely isolated. This phenomenon is considered by the concept of 'dynamic or growing capillarity envelope' which állows virtual bounding capillary pressure curves to be followed for any starting point of an imbibition process from the primary drainage capillary pressure curve.

Beattie [2] modeled cyclic steam 'stimulation processes and accounted for water-oil rélative permeability hysteresis by scanning between bounding drainage and imbibition curves. He üses a scanning curve exponent as history-match parameter in the simulation.

Tan [14] presented a rèvised formulation of the Killough model which reproduces the experimental bounding primary drainage capillary pressure curve for initialization of the reservoir.

For reasons of simplicity, the discussion which follows will consider gas or oil as always being a non-wetting (nw) phäse and brine a wetting phase for two phase hydrocarbon-brine systems. Oil will be considered a wetting phase with respect to gas and a nw phase relative to brinè. It is generally assumed that these conditions exist in the majority of sandstone petroleum producing reservoirs [5].

\section{THE MATHEMATICAL MODEL}

This chapter describes the mathematics and main principles behind the proposed dynamic hysteresis model for treating hysteresis in capillary pressure and relative permeabilities. To save space, the principles are figured out in a general way by using the following abbreviations:

$P_{c m}=$ Capillary pressúre between phases

$k_{n w}=$ Wetting phase $(\mathrm{w})$ relative permeability

$k_{m w}=$ Non wetting (nw) phase relative permeability

$S_{p}=$ Phase saturation.

where $m=g w$ or $m=o w$ for gas/water or oil/water systems and $m=g f$ for three phase gas/oil/water systems with $f=o+w$. $k_{r}$ and $P_{c}$ curves are presented as functions of the wetting phase saturation $(p=w)$.

Trapped Non-Wetting Phase Saturations: Land [10] found that flow properties of the drainage and imbibition systems differ because of the entrapment of the nw phase during imbibition (i). During imbibition, part of the nw phase is bypassed by the increasing wetting phase and leaves a portion of the niw phase in an immobile condition. As this trapped nw phase does not contribute to the flow, at a given saturation the $k_{r}$ to the nw phase is always less in the imbibition direction than in the drainage direction. Land found that the amount of entrapment at any saturation can be obtained from the relationship between initial nw phase saturation after a drainage process and residual saturation after complete imbibition. This relationship is a curve of characteristic shape and can be approximated by:

$$
\frac{1}{S_{n w r}}-\frac{1}{S_{n w i}}=C
$$

where the constant $C$ is a trapping characteristic of the porous rock. As soon as $C$ is determined from the experimental (d) and (i) functions, the trapped nw saturation left after (i) can be calculated for any maximum initial (or 'historical') nw phase saturation. Residual saturations increase with an increase in the initial saturations. : In our model we use the empirical relationship after Land for two phase gas/water or oil/water systems.

For three phase systems of water/oil/gas, both oil and gas are trapped as water saturation increases. Land assumed that the maximum residual hydrocarbon saturation is the same, regardless of whether the initial hydrocarbon saturation is gas, oil or both and that the trapped gas saturation for (i) can be calculated in the same manner as for the two phase cases, because the gas, being the nw phase, occupies the same pores regardless of the nature of the liquids present. Further he assumes that the difference of the reciprocals of the residuals and the initial total hydrocarbon saturations is a constant. Willhite [16] described the effect of trapped gas on residual oil saturation. He published a summation of experimental data and concluded that the residual oil saturation will be reduced if trapped gas is present at the, time when residual oil is trapped by water. This will improve recoveries in waterflooding. The published experimental data of Willhite do not support the theory of Land. Reduction in residual oil for an initial gas saturation is less than the amount of trapped gas, thus, the total residual hydrocarbon saturation is higher with additionally trapped gas. Therefore, for the three phase case we included a new treatment of the résiduals based on the publication of Willhite, because the Land approach may lead to unrealistic low residual oil saturations if initial and as a consequence residual gas saturation is high. This may lead to overestimation of oil recovery. Therefore, the model presented allows the input of a residual saturation function for both gas, and oil in dependence of the maximum historical gas saturation achieved prior the 
displacement by water. The residual saturation function for gas $(f)$ is given by:

$$
\begin{aligned}
& S_{g r}=f\left(S_{g i}\right) \\
& 0 \leq S_{g i} \leq 1 .-S_{L r} \text { where } S_{L r}=S_{w c}+S_{\text {org }}
\end{aligned}
$$

with the endpoint constraints that

$$
f\left(S_{g i}=S_{g c}\right)=S_{g c}
$$

and

$$
f\left(S_{g i}=\left(1 .-S_{L r}\right)\right)=S_{g r}^{M a x}
$$

The residual saturation function for oil $(F)$ is given by:

$$
S_{\text {orw }}=F\left(S_{g^{i}}\right)
$$

where

$$
F\left(S_{g i}=0\right)=S_{o r w}^{M a x}
$$

The function, which can be unique for each reservoir, should be determined experimentally and allows the control of the residual hydrocarbon saturations and therefore, the ultimate recovery of a reservoir.

Cyclic Drainage and Imbibition: Colonna [3] performed multi-cyclic two phase displacement experiments on two sandstone cores. He recognized that, at every point in a multi-cyclic displacement, the relationships among permeability, saturation and capillary pressure are a function of the past history of fluid movements at this point. He performed several cycles of primary drainage/imbibition/secondary drainage and recorded $P_{c}$ and $k_{r n w}$. In a first run he defined the conventional primary drainage (d) curve. A second run consisted of two (i) (dd) cycles, each starting from a different origin on the (d) curve (characterized by the maximum historical nw phase saturation) with an (i) process until nw phase saturation was isolated and a (dd) process back to the initial saturation for this loop. In a third experiment, the (d) curve was followed until maximum nw phase saturation, followed by a full (i) and (dd) cycle. For the graphical presentation of the obtained curves it is referred to the original paper. The conclusions which can be drawn from these experiments with respect to cyclic evolution of $P_{c}$ and $k_{r m}$ are:

1. The (d) curve has unique character and is obtained after an (i) and (dd) cycle starting from an original point on the (d) curve again.

2. Any complete (i) and (dd) cycle forms a closed loop, which means that the (dd) curve proceeds back to the original deviation point from the (d) curve.

3. Any closed loop, consisting of an (i) and (dd) curve is similar in shape regardless of the starting point on the (d) curve.

Our dynamic hysteresis model for $P_{c}$ and $k_{r}$ has been developed on the basis of the experimental observations after Colonna [3] and allows the reproduction of multi-cycle drainage-imbibition processes for capillary pressure, non- wetting and wetting phase relative permeabilities. The model takes into consideration that a primary drainage process can be stopped at any stage before the wetting phase is completely isolated, and an (i) process starts. It will be even possible to go through several (i) and (dd) cycles before wetting phase is completely isolated and (i) is starting.

Initialization of Reservoirs: Many theories of hydrocarbon accumulation exist. It is commonly assumed that the reservoir rock is initially water saturated and the hydrocarbons migrated into the formation reducing the water saturation [14]. This is by definition a primary drainage process (wetting phase is displaced by a nw phase). At that time when production starts, the saturation functions show already a history. Due to this history, reservoirs containing either gas or undersaturated oil, should be initialized with primary drainage capillary pressure curves, represented by an experimental curve obtained from a 100\% wetting phase saturated core by displacement with nw phase until wetting phase is isolated. For saturated oil reservoirs with a gas cap, it is assumed that the light and heavy hydrocarbons migrated into the trap together (primary drainage between brine and hydrocarbons) and the separation of gas and oil occurred within the trap (the nw gas phase displaces the oil from the gas cap volume - also a primary drainage process). Following these explanations, both two phase gas or undersaturated oil systems and three phase systems (gas/oil/water) are initialized with primary drainage (d) capillary pressure curves in our hysteresis model. For production or alternate production/injection processes (i) and (dd) curves are used. Using only one curve for both initialization and simulation causes an error in either determination of hydrocarbons in place or in simulation of production/injection cycles. Therefore, consideration of hysteresis in capillary pressure is recommended. For the non-hysteresis case, it is still free to give any input capillary pressure curve.

The Capillary Pressure Hysteresis Model: The capillary pressure hysteresis model has been developed based on the experimental observations of Colonna [3]. When starting to produce a reservoir, the $P_{c}$ functions show already a history. Following the previous explanations, the reservoir is initialized with the (d) curve. A (d) process can be stopped at any stage before wetting phase is completely isolated. Depending on the phase saturations after initialization and the following displacement process, the capillary pressure path to be followed can be different. After the theory of Colonna [3], a capillary pressure curve will be followed starting at the original initialization point on the (d) curve and ending up at the corresponding residual nw phase saturation for a complete (i) process. After a reversal in saturation change, a secondary drainage (dd) curve will be followed back until the original deviation point on the (d) curve is reached. This will be called the 'closed loop condition' for a complete (i) and (dd) cycle. There exist an infinite number of families of closed (i) (dd) hysteresis loops, each characterized by the deviation 
point from the (d) bounding curve and the corresponding residual (or trapped) nw phase saturation after a full (i) process (Fig. 1.1).

To account for this real dynamic hysteresis envelope behavior of $P_{c}$, the principal of 'scaling' of capillary pressure curves is applied. The model requires the input of maximum three curves based on experimental observations: 1. Primary drainage (d) $P_{c}$ curve, obtained by a displacement process from a $100 \%$ wetting phase saturated core by a nw phase until wetting phase is isolated. 2. Primary imbibition (i) $P_{c}$ curve, obtained by a further displacement by a wetting phase until nw phase is isolated. 3. Secondary drainage (dd) $P_{c}$ curve, obtained by a further displacement by a nw phase until residual wetting phase condition. These curves are further on called as 'original $P_{c}$ curves'. The original $P_{c}$ curves are converted into a normalized form. For this purpose, a normalized phase saturation is defined:

$$
S_{p}^{*}\left(S_{p}, S_{p}^{M i n}\right)=\frac{\left(S_{p}-S_{p}^{M i n}\right)}{\left(S_{p}^{M a x}-S_{p}^{M i n}\right)}
$$

where $S_{p}^{M a x}, S_{p}^{M i n}$ are the endpoint saturations of the respective original curves. The normalized original $P_{c}$ functions are defined as:

$$
P C^{*}\left(S_{p}\right)=\frac{P_{c}\left(S_{p}\right)-P_{c}\left(S_{p}^{M a x}\right)}{P_{c}\left(S_{p}^{M i n}\right)-P_{c}\left(S_{p}^{M a x}\right)}
$$

This normalizing procedure converts the original functions with given endpoint saturations and given extreme values of capillary pressure into normalized curves, called 'scaling functions', with values between zero and one vs. effective saturations also between zero and one. This procedure transforms the original $P_{c}\left(S_{p}\right)$ curves into normalized $P C^{*}\left(S_{p}^{*}\right)$ functions. The model allows (i) and (dd) curves to have negative values, too. Using the normalized capillary pressure functions enables to construct curves similar in shape for any other defined boundary saturation and capillary pressure endpoints (further on called 'scaling') by:

$$
\begin{aligned}
P_{c}\left(S_{p}\right) & =P_{c}\left(S_{p}^{\operatorname{Max}}\right) \\
+ & {\left[P_{c}\left(S_{p}^{M i n}\right)-P_{c}\left(S_{p}^{M a x}\right)\right] P C^{*}\left(\frac{S_{p}-S_{p}^{M i n}}{S_{p}^{M a x}-S_{p}^{M i n}}\right)^{(1.9)} }
\end{aligned}
$$

The derivative of the $P_{c}$ with respect to phase saturation required for the fully implicit calculation is given by:

$$
\begin{aligned}
\frac{\partial\left(P_{c}\right)}{\partial S_{p}}=\left[P_{c}\left(S_{p}^{M i n}\right)-P_{c}\left(S_{p}^{M a x}\right)\right] \\
\frac{1}{\left(S_{p}^{M a x}-S_{p}^{M i n}\right)} \frac{\partial\left[P C^{*}\left(S_{p}^{*}\right)\right]}{\partial S_{p}^{*}}
\end{aligned}
$$

Fig. 1.1 outlines the principal of scaling of (i) and (dd) loops for two different intermediate initial saturation conditions. Complete means, (i) until nw phase is isolated. For any maximum historical (or initial) nw phase saturation achieved prior an (i) process, the corresponding trapped nw phase saturation left after imbibition $\left(S_{n w w}\right)$ is calculated using Equ. 1.1. The lowest possible capillary pressure endpoint during imbibition $P_{c(i)}\left(S_{n w r}\right)$ at the residual nw phase saturation in the scaled loop is derived from the endpoint relationship of the original experimental loop by:

$$
\frac{P_{c(d)}\left(S_{p}^{M i n}\right)}{P_{c(d)}\left(S_{p}^{\text {Initial }}\right)}=\frac{P_{c(i)}\left(S_{n w r}^{M a x}\right)}{P_{c(i)}\left(S_{n w r}\right)}
$$

where $P_{d d)}\left(S_{p}^{M i x}\right)$ and $P_{\alpha(i)}\left(S_{n w r}{ }^{M a x}\right)$ are the endpoints of the experimental (d)/(i) curves and $P_{\alpha(d)}\left(S_{p}^{\text {litial }}\right)$ is the starting point capillary pressure for the actual loop.

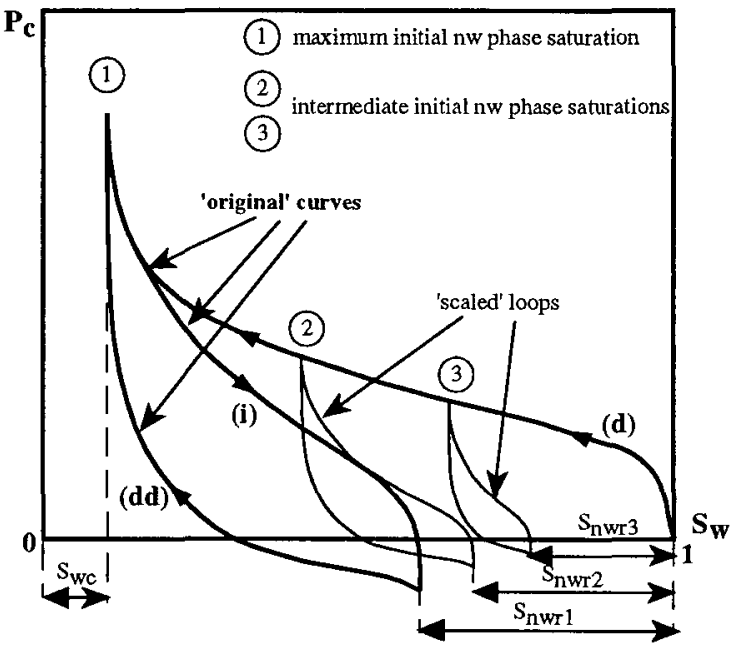

Figure 1.1: Original and 'intermediate' (i) and (dd) $P_{c}$ hysteresis loops

If the initial nw phase saturation in a block is less than the maximum one and drainage conditions continue, the (d) curve will be followed from the initialization point until a reversal in saturation change to (i) occurs. Such a process may occur e.g. at abandonment in an aquifer-gas storage reservoir complex when natural gas is injected. If after a complete (i) and (dd) cycle for an intermediate initial nw phase saturation drainage conditions continue and the historical nw phase saturation of the previous loop is exceeded, then the (d) curve will be followed until the next reversal to (i). This is called as the principle of 'growing hysteresis envelope'. Historical nw and its corresponding residual phase saturation is increasing at that time and fixed at the new reversal point. During cyclic displacements of wetting and nw phases, multiple saturation reversals may occur within (i) and (dd) loops, too. Our model allows such reversals both within the 'original' (i) and (dd) loop and within any 'scaled' hysteresis loop. For any reversal from (i) to (dd) within the loop, another curve will be scaled from the actual reversal point back to the initial point on the (d) curve. For reversals from a (dd) process to an (i) process, $P_{c}$ follows a scaled (i) curve to the actual $S_{n u r .}$. The treatment of $P_{c}$ for both possible reversals within a hysteresis loop is outlined in Fig.1.2: Starting from a fully wetting phase saturated medium at point [1], nw phase takes place during a (d) process (e.g. hydrocarbon accumulation). At an intermediate nw phase saturation at 
point [2], a reversal in saturation change occurs, and a scaled (i) curve is followed towards the actual residual nw phase saturation. Before the nw phase is trapped, another reversal to a (dd) process occurs [3] and a scaled curve from the actual reversal point to the original deviation point on the (d) curve will be followed. The treatment of any further reversal within the hysteresis loop is straightforward. If nw phase saturation exceeds the historical value (hysteresis loop is left at point [6]), $P_{c}$ follows the (d) curve until the next reversal in saturation change, where another hysteresis loop will be scaled.

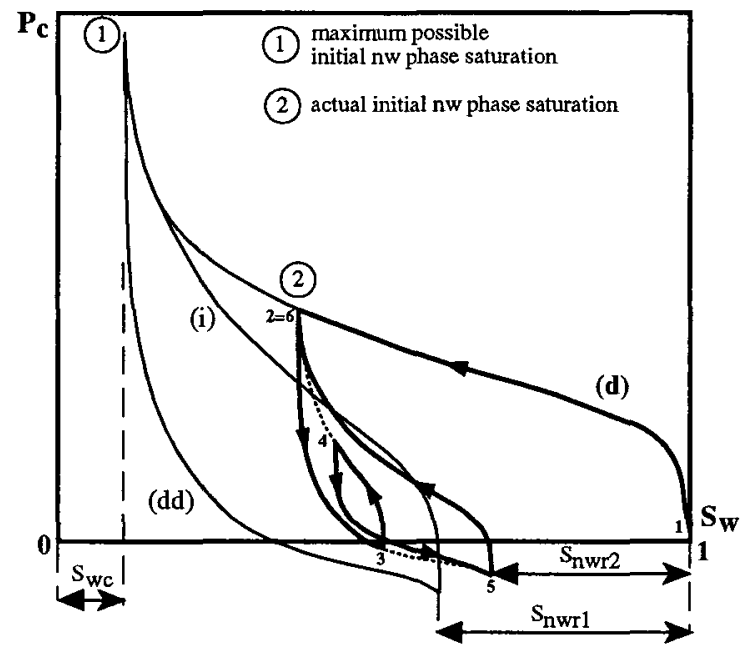

Figure 1.2: Treatment of multiple saturation reversals within $P_{c}$ hysteresis loop

The capillary pressure model presented allows to choose between different 'levels of hysteresis': 1 . Full treatment, with hysteresis both between (d), (i) and (dd) functions. 2 . Hysteresis only between (d) and (i) functions with the assumption that $(\mathbf{i})=(\mathrm{dd})$. This level is considered to be appropriate if only (i) processes are modeled. 3. Nonhysteresis case, with input of one curve, either (d) or (i) function. For the input of a (d) curve, the user must be aware, that initialization is correct, but an error is made during calculation of (i) processes. Using an (i) curve instead for both initialization and calculation results in an incorrect estimation of hydrocarbons in place.

The Relative Permeability Hysteresis Model: Several authors $[3,4,6,12]$ recognized hysteretic behavior of relative permeability functions during their experimental work. Again, like it is already apparent from capillary pressure curves, three sets of distinctly different curves, depending on the direction of saturation change and the past saturation history, has been observed experimentally for both wetting and nw phase relative permeabilities. Particularly the (d) and the (i) $k_{m w}$ curves are different because of the entrapment of the nw phase during (i). Colonna [3] concluded that hysteresis phenomena for $P_{c}$ and $k$, are obviously in analogy. For different intermediate nw phase saturations after a (d) displacement process he observed full $k_{r}$ hysteresis loops by an (i) process until residual nw phase saturation and a further (dd) process.
From his experiments the conclusions which can be drawn are in analogy to those for the $P_{c}$ cyclic experiments. The presented dynamic $k_{r}$ hysteresis model reproduces the experimental cyclic hysteretic behavior and allows accounting for hysteresis between (d) and (i) curves as well as between (i) and (dd) curves for both $k_{n w}$ and $k_{m w}$. Hysteretic relative permeability functions can be obtained experimentally.

The normalizing procedure for $k_{r}$ functions and the principle of 'scaling of functions' between endpoints is in analogy to those for the capillary pressure hysteresis model (Equ. 1.7 to Equ. 1.10) and therefore, not presented again. Again, the amount of entrapment after an (i) process is depending on the maximum historical nw phase saturation and will be calculated by Equ. 1.1. The treatment of the 'dynamic hysteresis envelope' behavior is in analogy to that of $P_{c}$ and is presented in Fig. 1.3 for $k_{m w}$.

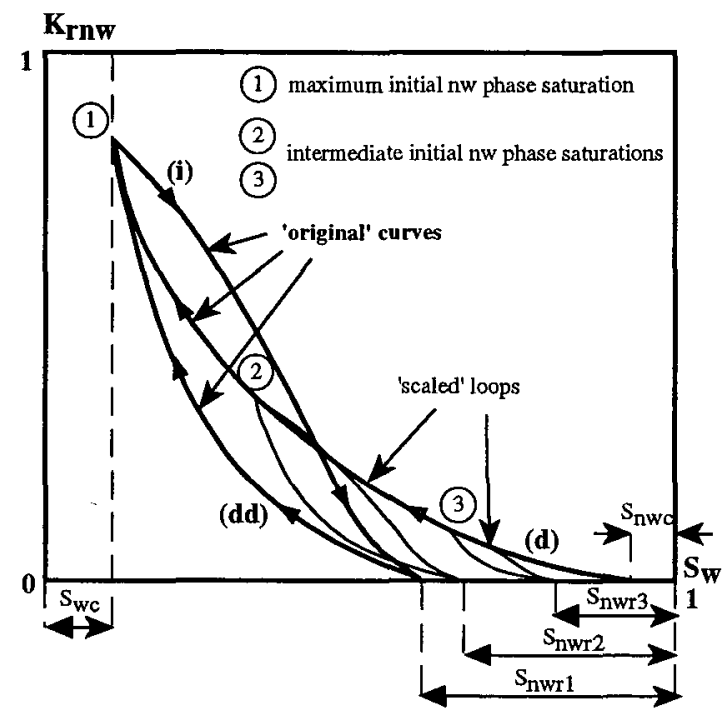

Figure 1.3: Original and 'scaled intermediate' (i) and (dd) $k_{m w}$ hysteresis loops

Due to the similarity in shape of experimental $k_{r}$ hysteresis loops for different historical nw phase saturations, a procedure of 'scaling' of curves is applied for (i) and (dd) hysteresis loops again. The loops are scaled between the point on the (d) curve at any intermediate initial nw phase saturation and between zero $k_{r}$ at the trapped nw phase saturation.

Multiple saturation reversals within original and scaled loops are allowed too, as outlined in Fig. 1.4: The saturation history outlined is starting with a (d) process [1-2], followed by an (i) process like it is during production. A scaled (i) curve towards zero $k_{m w}$ at the actual $S_{n w r 2}$ is followed until a further saturation reversal to (dd) at point [3] stops the (i) process. Another scaled (dd) curve follows back to the original deviation point on the $(d)$ curve (point $[2=6]$ ), but is also interrupted by a reversal to (i). It is assumed that nw phase saturation achieves the trapped state and $k_{m w}$ becomes zero at point [5]. For the following (dd) process the bounding curve of the hysteresis loop is followed back until at point [6] the (d) curve is reached and the 
experimental closed loop condition (after Colonna [3]) is fulfilled. If drainage conditions continue, the original (d) curve is followed until the next saturation reversal with a new scaled hysteresis loop. If maximum nw phase saturation is achieved prior (i), the 'original' curves are reproduced.

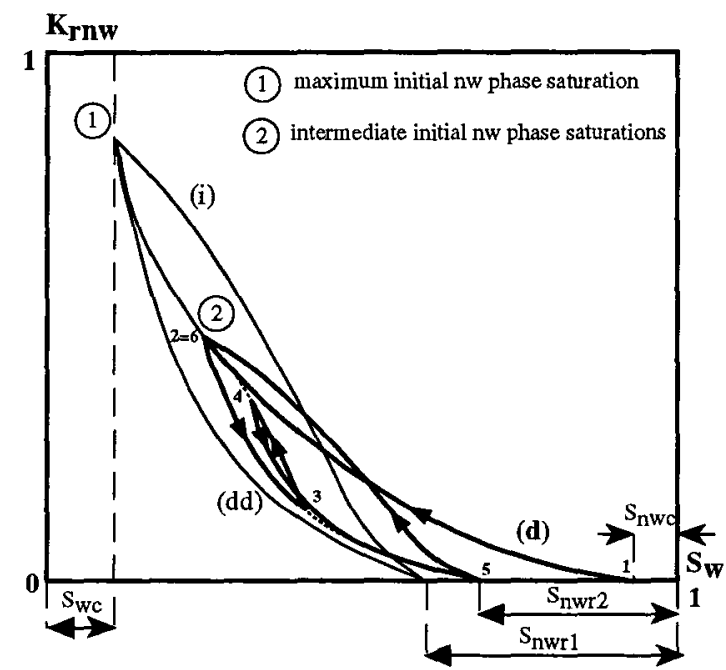

Figure 1.4: Treatment of multiple saturation reversals within $k_{m w}$ hysteresis loop

Wetting phase permeability hysteresis is somewhat different in behavior, especially with respect to endpoint treatment, and therefore discussed separately. For water wet systems, the trapped water saturation has relatively little effect on the nw relative permeability. But when e.g. oil is at its residual saturation, it is effectively blocking the flow of water in the pores, thus causing a significant reduction in $k_{r w}$. Typically, water endpoint permeabilities are between 0.05 and 0.3 at residual oil saturations of about $25 \%$ for oil/water systems [6].

In our model, hysteresis between (d), (i) and (dd) $k_{n w}$ functions is fully accounted for. A (d) process (hydrocarbon accumulation or injection like in a aquifer gas storage reservoir) can be stopped at any intermediate nw phase saturation followed by an (i) process. The principle of scaled intermediate hysteresis loops is applied on $k_{m w}$, too and is presented in Fig. 1.5. (i) $k_{r w}$ curves are scaled between the origin at the (d) curve at the maximum historical nw phase saturation and an interpolated endpoint at the corresponding residual nw phase saturation. The interpolated maximum $k_{n w}$ at $S_{n w r}$ is obtained by linear interpolation between the upper endpoint of the 'original' experimental (i) curve at maximum residual nw phase saturation and the upper endpoint of the experimental (d) curve at $100 \%$ wetting phase saturation; as outlined in Fig. 1.5 for two hysteresis loops. Linear interpolation was used because there were no experimental data available that allows a non-linear approximation between the endpoints.

Handling of multiple reversals in saturation change within original and scaled hysteresis loops is in analogy as presented for $k_{m w}$ and therefore not presented again. If nw phase saturation becomes less than the residual one during an (i) process (e.g. residual gas may be compressed and/or dissolved in the oil when pressure increases), then the interpolated linear connecting curve towards maximum primary drainage (d) $k_{n w}$ endpoint value is followed until a reversal in saturation change occurs. For the following (dd) process a scaled curve is followed back to the original deviation point on the (d) curve.

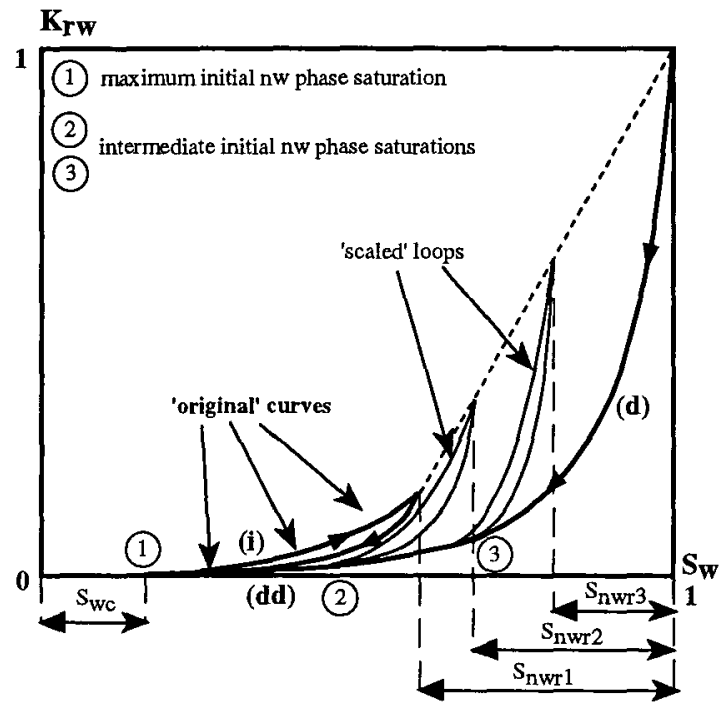

Figure 1.5: Original and 'scaled intermediate' (i) and (dd) $k_{r w}$ hysteresis loops

In the presented hysteresis model, the actual path of a saturation function to be followed during cyclic displacement processes is determined by remembering the history in saturation. The maximum historical nw phase saturation is determining the residual ones. This leads to more realistically predictions of fluid flow behavior within transition zones or in zones with cyclic nw phase saturation changes (like in an aquifer-reservoir complex at abandonment).

The flexible treatment of the endpoints of saturation functions together with the 'scaling' of curves between them is reflected in the different flow behavior and ultimate recovery in comparison to the non-hysteresis case.

The 'Level of Hysteresis' Option: The presented hysteresis model allows to chose between different 'levels of hysteresis'. Hysteresis can be accounted for $P_{c}$ functions only or for $P_{c}$ and $k_{r}$ functions together. For both sets of functions one may choose between full hysteresis (hysteresis between (d)/(i) and (i)/(dd) functions) or hysteresis between (d)/(i) functions only, which means that (i) and (dd) functions are assumed to be equal.

With the 'level of hysteresis' option for saturation functions only those input curves must be given that reflect the dominant displacement process in a simulation project. Obviously, for accurate prediction of the reservoir behavior, the selected set of curves should be in accordance with the expected directions of saturation change. 
Endpoint Scaling for Rock Regions: Normalized saturation functions are used to create capillary pressure and relative permeability functions for those rock regions with known endpoints only, by relating them to rock regions with known experimental curves. This method is called 'endpoints scaling', because experimental (or analytical) curves with given endpoints in saturation and function value are 'scaled' to different endpoints by retaining their shape. This is a very useful option for those reservoirs, where saturation functions behave similar, but endpoints vary greatly throughout the reservoir extension. Saturation functions obtained by laboratory work can be averaged and given in tabular form. For any other rock region, only the changing endpoints must be given as input. This option can be used also as a tool for history matching by endpoint variation.

Three Phase Relative Permeability: The hysteresis model uses the calculation of three phase $k_{r}$ after Stone [13]. The Stone 1 and Stone 2 models predicts three phase $k_{r}$ by using the two phase gas/oil and oil/water $k_{r}$. Relative permeabilities are calculated first independently for the two phase systems, accounting for the effects of hysteresis. The obtained values are combined in the Stone's models for calculating of the three phase oil relative permeability. Therefore, the effects of hysteresis are reflected from the Stone's three phase $k_{r}$ model, too. For the Stone's equations it is referred to the original paper [13].

\section{VALIDATION OF THE HYSTERESIS MODEL}

The validation part of the paper focuses on the presentation of the major effects when accounting for the hysteretic behavior of saturation functions in comparison to the non-hysteresis case using simplified functions. There are three main effects to be demonstrated by the validation:

1. Influence of historical on residual nw phase saturation together with its influence on recoverable hydrocarbons for two and three phase cases

2. Difference in the phase quantities moved with time during displacement with and without considering the hysteretic behavior of saturation functions

3. Effect of initialization on the recoverable fluid quantities

In order to focus on the effects listed above and to avoid the influence of other simulation parameters, it was decided to use hypothetical examples in one dimension for validation. The hysteresis model is limited by the constraint that for those parts of the simulations where the actual displacement process corresponds to that assumed using simplified saturation functions of the non-hysteresis case, the hysteresis model should give identical results.

Transition Zone Example: For simulating the influence of maximum historical nw phase saturation on residual one and therefore on movable fluid quantities, a linear vertical oil/water example was set up. The model was filled originally with undersaturated oil and water, distributed vertically due to the equilibrium between gravity and capillary forces, with the first block at the top at $S_{o}^{\text {Max }}$ and connate water saturation and the last block at the bottom filled $100 \%$ with water, thus representing the aquifer together with a water injection well. A (d) $P_{c}$ curve (Table A-2) was used for initialization. Fig. 1.6 shows the vertical saturation distribution after initialization and after producing nearly $100 \%$ of the movable oil with and without accounting for hysteresis in relative permeabilities. In accordance with the Land equation (Equ. 1.1) the $S_{o r}$ for the hysteresis case is ranging between the $S_{o r}$ ax at the top (at initial $S_{o}^{M a x}$ ) and zero at the bottom (initial water saturated block) throughout the vertical extension compared with the constant maximum $S_{o r}$ for the non-hysteresis case. The 'amount' of additional oil recoverable by effect of hysteresis is also outlined in Fig. 1.6. $k_{r}$ data with hysteresis are taken from reference 6 and given in Table A-1.

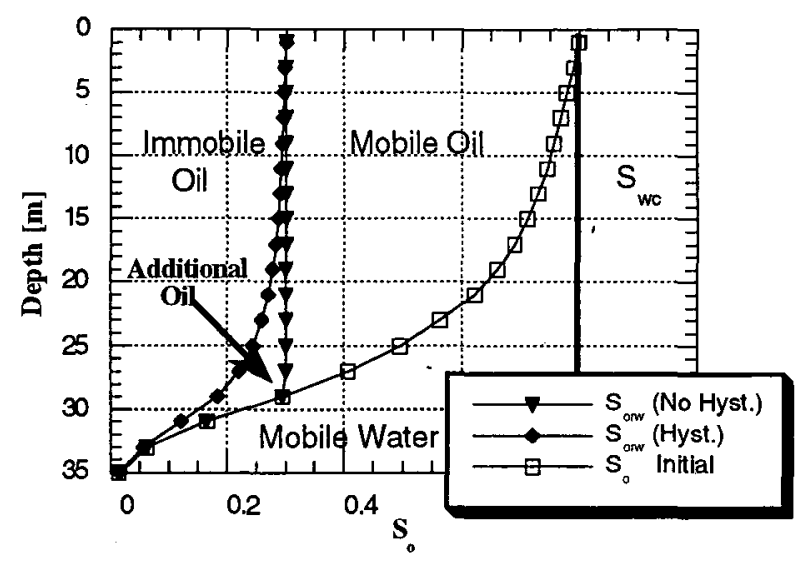

Figure 1.6: Initial and residual vertical saturation distribution for a bottom water drive example

The dependence of the residual nw phase saturation on the initial one will lead to a significant higher cumulative production for those reservoirs which have extended transition zones. Fig. 1.7 outlines that the difference in ultimate oil recovery for the simulated transition zone reservoir is nearly $10 \%$ higher for the hysteresis case in $k_{r}$.

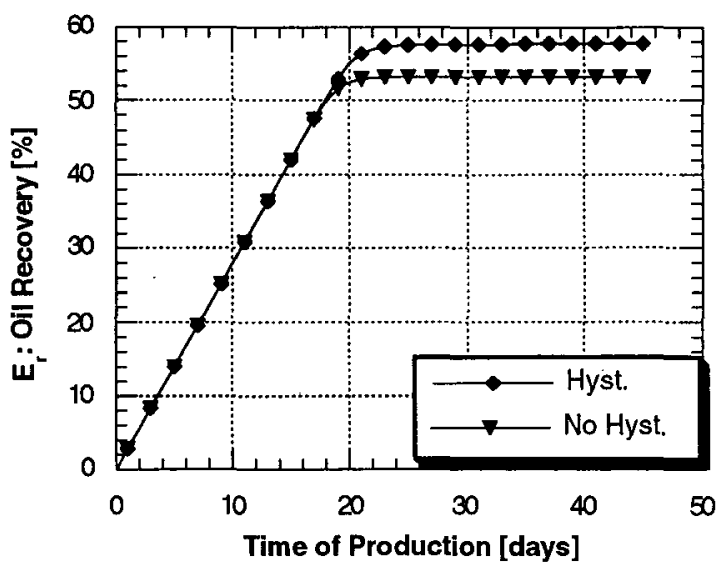

Figure 1.7: Oil recovery from a transition zone reservoir: Influence of hysteresis in $k_{r}$ 
The reservoir was initialized with a given drainage capillary pressure curve (Table A-2). The recovery curve is nearly identical in shape for both the hysteresis and non hysteresis case, because the actual process is an (i) one and for the non-hysteresis case (i) curves are used, too.

Gas Storage Example: During gas storage operations, cyclic drainage and imbibition displacement processes take place. In order to investigate the effect of hysteresis in saturation functions on the gas storage reservoir behavior under water drive mechanism, a linear vertical example was set up. The gas injection/production well was located at the top, the water drive mechanism is represented by a water production/injection well with constant pressure at the bottom. The model was initially saturated $100 \%$ with water, thus representing a gas storage reservoir at abandonment or an aquifer gas storage reservoir itself. Data of $k_{r}$ were taken from reference 4 (Table A-3). $P_{c}$ functions with hysteresis are based on our own experimental work (Table A-4).

We simulated one complete injection and production cycle with a shut in period of one month between, which represents a typical cycle in gas storage operations over one year. The sensitivity of the cumulative injection/production on the different set of saturation functions used was investigated.

$k_{r}$ data from our own experimental observations were used, too. As the $k_{r}$ data from reference [4] gave the more representative results, they are presented here. For the hysteresis case, a set of (d) functions, representing a displacement process of water by gas during injection into the aquifer, and (i) functions, representing the production of the gas, were given. The cumulative injection/production rate was observed for the hysteresis case and compared with those results using either (d) or (i) curves for both displacement directions in the non-hysteresis case.

Fig. 1.8 outlines on the one hand that using single (d) curves, the reservoir is reproducing the injection behavior of the hysteresis case (by definition, because the hysteresis case is also using a (d) curve for injection into the aquifer), but the production is significantly overestimated, because the used $k_{r g d}$ curve becomes zero only at $S_{g r}=S_{g c}$, which is a really unrealistically approach and leads to nearly $100 \%$ recoverable gas, if $S_{g c}$ is very small or zero.

Using (i) curves for the non-hysteresis case on the other hand, reproduces the same production curves (by definition, because the hysteresis case is also using an (i) curve for production) but leads to an overestimation of injectivity in comparison to the hysteresis case. This is mainly due to the fact, that $k_{r w i}$ curves were higher in value than $k_{r w d}$ curves and additionally linear extrapolated to the water saturation values of 1 , thus making the water easier to be displaced by the gas.

A separate run showed that the influence of $P_{c}$ hysteresis (Data from Table A-4) is minor for this model and set of input curves in comparison to the effects of $k_{r}$ hysteresis.

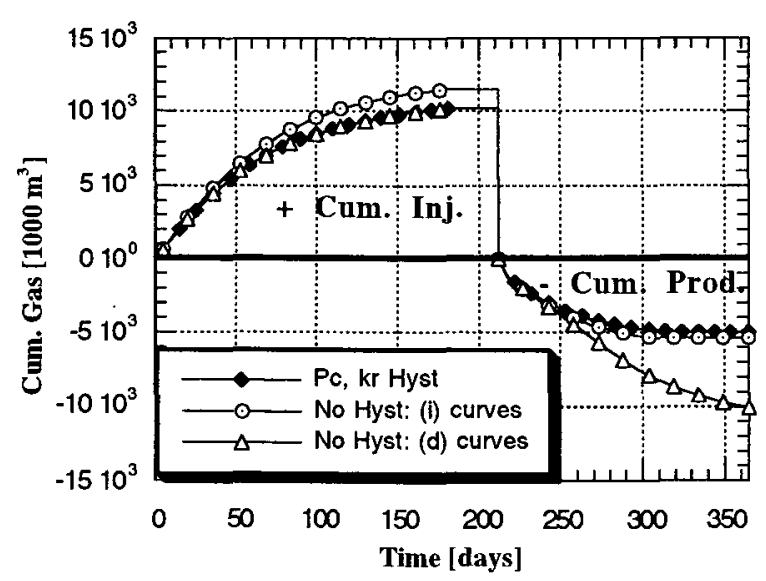

Figure 1.8: Cumulative injection/production of gas during a one year gas storage cycle: Effect of hysteresis

It can be concluded from this simulation example, that for the simulation of cyclic gas storage operations, especially near the gas/water transition zone and in aquifer storage reservoirs, for realistic predictions of reservoir performance in both displacement directions, hysteresis in saturation functions must be accounted for.

Waterflooding in the Presence of Free Gas: Waterfloods often start when reservoir pressure has already declined and there is an appreciable free gas saturation in the pore space. When water is injected into the reservoir containing oil and gas, residual saturations of both may remain. Willhite [16] presented a summation of experimental data about the reduction of residual oil saturation with an initial free gas present when starting the waterflood. Both $S_{g r}$ and $S_{\text {or }}$ are now functions of $S_{g i}$

For this example we used the residual gas and oil saturation data (Table A-5) after Willhite. He presented $S_{g r}$ and $\Delta S_{o r}$ as functions of $S_{g i}$. For investigating the effect of trapped gas on residual oil during waterfloods in solution gas-drive reservoirs, a linear vertical example was set up. $k_{r}$ and $P_{c}$ data are given in Tables A-6, A-7, A-8. The model was assumed to have already a history of production of oil under solution gas drive. Various initial free gas saturations were established beside the oil. Water saturation was initially that of connate water.

During the following waterflood, the cumulative oil production between the hysteresis case (taking into account the $\Delta S_{o r}$ reduction for the initial $S_{g i}$ ) and the non-hysteresis case (considering constant $S_{\text {or }}$ in the given rock region) was compared (Fig. 1.9 and 1.10). The actual recovered oil at a certain time $(Q)$ after starting to flood is presented as percentage of total movable oil considering the fixed $S_{o r}$ condition of the non-hysteresis case $\left(Q^{*}\right)$. Fig. 1.9 outlines that for an initial free gas saturation of only $5 \%$ the recovery without accounting for the effects of $\Delta S_{\text {or }}$ reduction is underestimated by about $7 \%$, for $15 \%$ initial gas by about $20 \%$ (Fig. 1.10). Additionally, the accounting of hysteresis results in higher values of $k_{r o}$, which is due to the scaling of the $k_{\text {rogi }}$ function, thus making the oil more mobile and leading to faster recovery (Fig. 1.9 and 
Fig. 1.10). Obviously, the ultimate recovery strongly depends on the introduced residual saturation function, which becomes now a tool. The function is unique for each reservoir and should be observed experimentally.

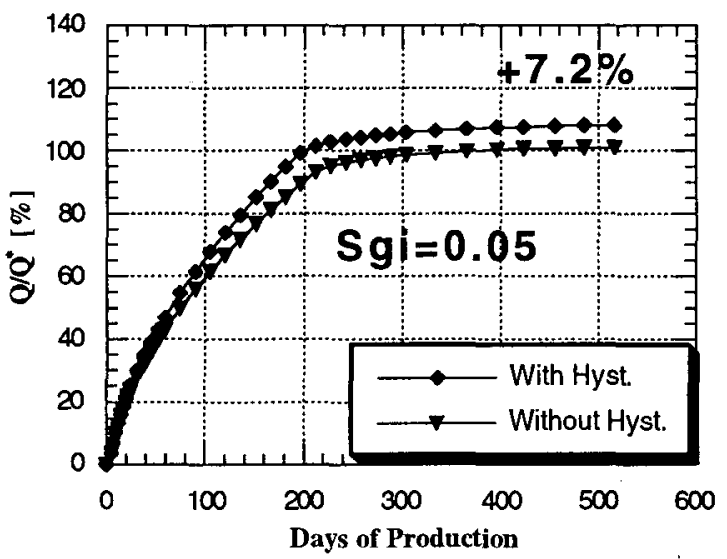

Figure 1.9: Waterflooding in the presence of free gas: Effect of initial free gas saturation on recovery $\left(S_{g}=0.05\right)$

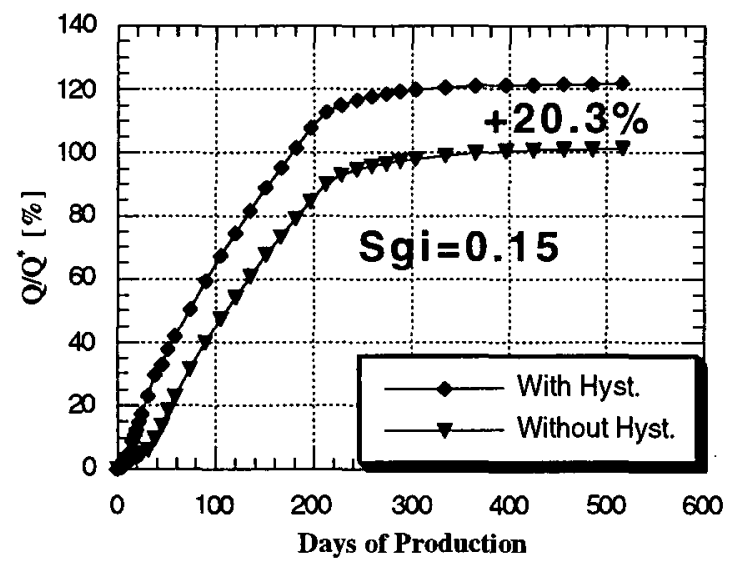

Figure 1.10: Waterflooding in the presence of free gas: Effect of initial free gas saturation on recovery $\left(S_{g}=0.15\right)$

\section{CONCLUSIONS}

A new hysteresis model for saturation functions has been developed and incorporated in a 3D, three phase, fully implicit reservoir simulator. The main principles applied are based on both published and own experimental observation of hysteresis phenomena in capillary pressure and relative permeabilities. The parametric study on hypothetical reservoirs leads us to the following conclusions:

- Realistic reservoir predictions of displacements processes with changing directions in saturation change requires the consideration of hysteresis in saturation functions.

- Hydrocarbon reservoirs should be initialized with primary drainage $P_{c}$ curves, for production or alternate production/injection the effect of hysteresis should be accounted for by using (i) and (dd) $P_{c}$ curves.

- The influence of maximum historical on residual nw phase saturation must be accounted for realistically predictions of flow behavior if initial nw phase saturation is different from the maximum one (e.g. within transition zones).

- The effect of trapped gas on residual oil saturation for waterflooding in the presence of free gas is significantly influencing the recovery and must be accounted for.

- The additional storage requirement for the hysteresis case is low, because for remembering the history of a block only the value of maximum historical nw phase saturation and the actual direction of saturation change has to be stored. Computation time is only slightly increased by using hysteresis.

- Although the principles behind the model are rather clear, the model is difficult to programme and care must be taken with respect to stability.

- The 'level of hysteresis' option allows to select the degree of complexity required for a specific simulation project.

- The 'scaling of endpoints' option allows to define saturation function by giving endpoints and relating them to similar functions with known endpoints and function shape.

\section{NOMENCLATURE}

$P_{c m}=$ Capillary pressure between phases

$k_{r w}=$ Wetting phase $(\mathrm{w})$ relative permeability

$k_{r m w}=$ Non wetting (nw) phase relative permeability

$S_{p}=$ phase saturation

$S_{p}^{*}=$ normalized phase saturation

$P C^{*}=$ normalized capillary pressure function

(d) = primary drainage curve/process

(i) = imbibition curve/process

(dd) $=$ secondary drainage curve/process

$\mathrm{w}=$ wetting phase

nw $=$ non-wetting phase

$\phi \quad=$ porosity []

$\mathbf{k}=$ permeability [md]

$\mu=\operatorname{viscosity~[cp]~}$

$\mathrm{B}$ = formation volume factor []

$\mathrm{R}_{\mathrm{s}}=$ solution gas ration []

$\mathrm{E}_{\mathrm{r}}=$ Recovery [\%]

$\mathrm{Q}=$ Cumulative production or injection $\left[\mathrm{m}^{3}\right]$ 


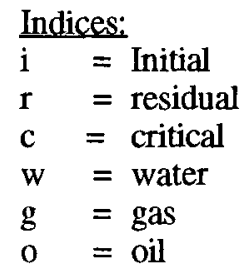

\section{Superscripts:}

Max $=$ maximum

Min $=$ minimum

\section{CONVERSION FACTORS}

$\begin{array}{lrlr}{[\mathrm{bar}]} & \mathrm{x} & 1.450377 \mathrm{E}+01= & {[\mathrm{psi}]} \\ {[\mathrm{m} 3 / \mathrm{day}] \mathrm{x}} & 6.289811= & {[\mathrm{STB} / \mathrm{day}]} \\ {\left[\mathrm{m}^{2}\right]} & \mathrm{x} & 1.0132503 \mathrm{E}+09= & {[\mathrm{md}]} \\ {[\mathrm{m}]} & \mathrm{x} & 3.280840= & {[\mathrm{ft}]} \\ {[\mathrm{Pa} \mathrm{s}]} & \mathrm{x} & 1.0 \mathrm{E}+03= & {[\mathrm{cp}]}\end{array}$

\section{ACKNOWLEDGMENT}

I like to thank Mr. K. Potsch and Mr. N. Philippowitsch from OMV for their contribution in the experimental work on the hysteresis model and the staff of the reservoir engineering department for its support during the work on this project.

\section{REFERENCES}

1. Bang, H. W.: "Simulation Study Shows Hysteresis Effect on Oil Recovery During a Cyclic Steam Process", Oil \& Gas J. (February 27, 1984), pp 83-86

2. Beattie, C.I.; Boberg, T.C.; McNab, G.S.: "Reservoir Simulation of Cyclic Steam Stimulation in the Cold Lake Oil Sands", paper SPE 18752 presented at the SPE California Regional Meeting, Bakersfield, California, April 5-7, 1989

3. Colonna, J.; Brissaud, F.; Millet, J.L.: "Evolution of Capillarity and Relative Permeability Hysteresis", Soc. of Petr. Eng. J. (Feb. 1972), pp 28-38; Trans., AIME (1972) Vol. 253

4. Evrenos, A. I.; Comer, A.G.: "Numerical Simulation of Hysteretic Flow in Porous Media", paper SPE 2693 presented at the SPE-AIME 44th Annual Fall Meeting, Denver, Colorado, Sept. 28 - Oct. 1, 1969

5. Geffen, T.M.; Owens, W.W.; Parrish, D.R.; Morse, R.A.: "Experimental Investigation of Factors Affecting Laboratory Relative Permeability Measurements", Trans., AIME (1951) Vol. 192, pp 99-110

6. Hagoort, J.: "Measurement of Relative Permeability for Computer Modeling/Reservoir Simulation", Oil \& Gas J. (February 20, 1984), pp 62-68
7. Holgren, C.R.; Morse, R.A.: "Effect of Free Gas Saturation on Oil Recovery by Water Flooding", Trans., AIME (1951) Vol. 192, pp 135-140

8. Killough, J.E.: "Reservoir Simulation With History-Dependent Saturation Functions", Soc. of Petr. Eng. J. (February 1976), pp 37-48

9. Kyte, J.R.; Stanclift, R.J.; Stephan, S.C.; Rapoport, L.A.: "Mechanism of Water Flooding in the Presence of Free Gas”, Trans., AIME (1956) Vol. 207, pp 215-221

10. Land, C. S.: "Calculation of Imbibition Relative Permeability for Two- and Three-Phase Flow From Rock Properties", Soc. of Petr. Eng. J. (June 1968), pp 149-156; Trans., AIME (1968) Vol. 243

11. Land, C. S.: "Comparison of Calculated with Experimental Imbibition Relative Permeability", Trans., AIME (1971) Vol. 251, pp 419-425

12. Osoba, J.S.; Richardson, J.G.; Kerver, J.K.;Hafford, J.A.; Blair, P.M.: "Laboratory Measurements of Relative Permeability”, Trans., AIME (1951) Vol. 192, pp 47-56

13. Stone, H.L.: "Probability Model for Estimating Three-Phase Relative Permeability", JPT (Feb. 1970), pp 214-218, Trans., AIME (1970) Vol. 249

14. Tan, T.: "Representation of Hysteresis in capillary pressure for Reservoir Simulation Models", J. of Can. Petr. Techn. (July-August 1990) Vol. 29, No 4, pp 85-88

15. Wei, Jun-Zhi; Lile, O. B.: "Influence of Wettability and Saturation Sequence on Relative Permeability Hysteresis in Unconsolidated Porous Media", SPE 25282, Unsolicited

16. Willhite, P. G.: "Waterflooding”, SPE Textbook Series, Richardson, TX, 1986

\section{Appendix A: EXAMPLE DESCRIPTIONS}

Pressures in Tables A-2, A-4, A-8 and A-9 are given in [psi].

\section{Transition Zone Example}

Vertical linear model: $20 \times 1 \times 1$ blocks ( $2 \times 30 \times 30$ meter) $\phi=0.20 ; \mathrm{k}=1,000 \mathrm{md}$; production with $\mathrm{p}_{\mathrm{wf}}=150$ bar, injection with $\mathrm{q}=100 \mathrm{~m}^{3} /$ day

Table A-1: Oil/water $\mathrm{k}_{\mathrm{r}}$ data with hysteresis

\begin{tabular}{|lllllll|}
\hline Sw & krwd. & kwi & krwdd & krowd & krowi & krowdd \\
\hline 0.200 & 0.000 & 0.000 & 0.000 & 0.720 & 0.720 & 0.720 \\
0.300 & 0.005 & 0.010 & 0.005 & 0.420 & 0.560 & 0.350 \\
0.400 & 0.020 & 0.040 & 0.020 & 0.290 & 0.350 & 0.180 \\
0.500 & 0.060 & 0.080 & 0.060 & 0.200 & 0.170 & 0.090 \\
0.600 & 0.115 & 0.125 & 0.120 & 0.125 & 0.050 & 0.030 \\
0.700 & 0.222 & 0.170 & 0.170 & 0.084 & 0.000 & 0.000 \\
0.800 & 0.420 & & & 0.040 & & \\
0.900 & 0.665 & & & 0.020 & & \\
1.000 & 1.000 & & & 0.000 & & \\
\hline
\end{tabular}


Table A-2: Oil/water $P_{c}$ data with hysteresis

\begin{tabular}{|llll|}
\hline Sw & Pcow & Pcowi & Pcowdd \\
\hline 0.2000 & 7.000 & 7.000 & 7.0000 \\
0.260 & 4.800 & 3.400 & 4.8000 \\
0.300 & 4.000 & 2.000 & 3.6000 \\
0.350 & 3.300 & 1.300 & 2.8000 \\
0.400 & 2.900 & 0.900 & 2.3000 \\
0.500 & 2.300 & 0.500 & 1.5500 \\
0.600 & 1.850 & 0.200 & 0.9000 \\
0.700 & 1.500 & 0.000 & 0.0000 \\
0.800 & 1.200 & & \\
0.900 & 0.900 & & \\
0.990 & 0.400 & & \\
1.000 & 0.000 & & \\
\hline
\end{tabular}

\section{Gas Storage Example}

Vertical linear model: $20 \times 1 \times 1$ blocks (10x50x50 meter) $\phi=0.24 ; \mathrm{k}=500 \mathrm{md}$; production and injection to maintain constant pressure gradient in the model.

Table A-3: Gas/water $\mathbf{k}_{\mathrm{T}}$ data with hysteresis

\begin{tabular}{|lllllll|}
\hline Sw & krwd & krwi & krwdd & krgd & krgi & krgdd \\
\hline 0.300 & 0.000 & 0.000 & 0.000 & 0.580 & 0.580 & 0.580 \\
0.350 & 0.002 & 0.004 & 0.010 & 0.420 & 0.545 & 0.330 \\
0.400 & 0.006 & 0.015 & 0.025 & 0.330 & 0.480 & 0.200 \\
0.450 & 0.011 & 0.040 & 0.060 & 0.220 & 0.370 & 0.120 \\
0.500 & 0.022 & 0.080 & 0.140 & 0.160 & 0.170 & 0.060 \\
0.550 & 0.046 & 0.160 & 0.250 & 0.120 & 0.070 & 0.030 \\
0.600 & 0.080 & 0.250 & 0.340 & 0.094 & 0.020 & 0.010 \\
0.650 & 0.135 & 0.400 & 0.400 & 0.070 & 0.000 & 0.000 \\
0.700 & 0.200 & & & 0.050 & & \\
0.750 & 0.300 & & & 0.035 & & \\
0.800 & 0.410 & & & 0.025 & & \\
0.850 & 0.540 & & & 0.015 & & \\
0.900 & 0.680 & & & 0.010 & & \\
0.950 & 0.840 & & & 0.003 & & \\
1.000 & 1.000 & & & 0.000 & & \\
\hline
\end{tabular}

Table A-4: Gas/water $P_{c}$ data with hysteresis

\begin{tabular}{|llll|}
\hline Sw & Pcgwd & Pcgwi & Pcgwdd \\
\hline 0.30 & 116.03 & 116.03 & 116.03 \\
0.33 & 66.72 & 15.95 & 37.71 \\
0.40 & 29.01 & 2.61 & 10.15 \\
0.50 & 14.50 & 0.58 & 5.51 \\
0.60 & 7.25 & 0.15 & 2.18 \\
0.65 & 5.80 & 0.00 & 0.00 \\
0.70 & 4.79 & & \\
0.80 & 3.77 & & \\
0.90 & 3.05 & & \\
1.00 & 0.00 & & \\
\hline
\end{tabular}

\section{Waterflooding Example}

Vertical linear model: $1 \times 1 \times 20$ blocks ( $30 \times 50 \times 30$ meter) $\phi=0.20 ; \mathrm{k}=1,000 \mathrm{md}$; production with $\mathrm{p}_{\mathrm{wf}}=150 \mathrm{bar}$, injection with $\mathrm{q}=1000 \mathrm{~m}^{3} /$ day

Table A-5: Residual saturation functions

\begin{tabular}{|lll|}
\hline Sgi & Sgr & Sor \\
\hline 0.000 & 0.000 & 0.350 \\
0.040 & 0.040 & 0.325 \\
0.100 & 0.080 & 0.300 \\
0.150 & 0.110 & 0.282 \\
0.200 & 0.140 & 0.270 \\
0.300 & 0.195 & 0.250 \\
0.400 & 0.238 & 0.235 \\
0.430 & 0.250 & 0.230 \\
\hline
\end{tabular}

Table A-6: Oil/water $\mathrm{k}_{\mathrm{r}}$ data with hysteresis

\begin{tabular}{|lllllll|}
\hline Sw & krwd & krwi & krwdd & krowd & krowi & krowdd \\
\hline 0.220 & 0.000 & 0.000 & 0.000 & 1.000 & 1.000 & 1.000 \\
0.250 & 0.004 & 0.007 & 0.005 & 0.700 & 0.830 & 0.400 \\
0.300 & 0.010 & 0.017 & 0.014 & 0.350 & 0.550 & 0.160 \\
0.350 & 0.018 & 0.030 & 0.024 & 0.200 & 0.280 & 0.095 \\
0.400 & 0.023 & 0.045 & 0.035 & 0.160 & 0.125 & 0.050 \\
0.450 & 0.033 & 0.065 & 0.050 & 0.130 & 0.070 & 0.030 \\
0.500 & 0.045 & 0.090 & 0.065 & 0.100 & 0.040 & 0.015 \\
0.550 & 0.060 & 0.120 & 0.090 & 0.080 & 0.020 & 0.008 \\
0.600 & 0.080 & 0.150 & 0.130 & 0.065 & 0.010 & 0.002 \\
0.650 & 0.105 & 0.200 & 0.200 & 0.050 & 0.000 & 0.000 \\
0.700 & 0.155 & & & 0.040 & & \\
0.800 & 0.320 & & & 0.020 & & \\
0.900 & 0.600 & & & 0.010 & & \\
1.000 & 1.000 & & & 0.000 & & \\
\hline
\end{tabular}

Table A-7: Gas/oil $\mathrm{k}_{\mathrm{z}}$ data with hysteresis

\begin{tabular}{|lllllll|}
\hline $\mathrm{S}_{\mathbf{L}}$ & krogd & krogi & krogdd & krgd & krgi & krgdd \\
\hline 1.000 & 1.000 & & & & & \\
0.980 & 0.750 & & & & & \\
0.960 & 0.600 & & & 0.000 & & \\
0.940 & 0.500 & & & 0.010 & & \\
0.850 & 0.200 & & & 0.050 & & \\
0.800 & 0.100 & & & 0.080 & & \\
0.750 & 0.050 & 0.200 & 0.200 & 0.115 & 0.000 & 0.000 \\
0.740 & 0.044 & 0.160 & 0.190 & 0.120 & 0.120 & 0.010 \\
0.720 & 0.030 & 0.110 & 0.160 & 0.135 & 0.160 & 0.020 \\
0.670 & 0.015 & 0.055 & 0.100 & 0.175 & 0.240 & 0.055 \\
0.620 & 0.007 & 0.020 & 0.050 & 0.235 & 0.290 & 0.140 \\
0.600 & 0.005 & 0.010 & 0.030 & 0.260 & 0.305 & 0.210 \\
0.570 & 0.000 & 0.000 & 0.000 & 0.320 & 0.320 & 0.320 \\
\hline
\end{tabular}

Table A-8: $P_{c}$ data with hysteresis

\begin{tabular}{|cccccccc|}
\hline Sw & Pcogd & Pcogi & Pcogdd $^{\circ}$ & $\mathrm{s}_{\boldsymbol{f}}$ & Pcgfd & Pcgfi & Pcgfdd \\
\hline 0.22 & 7.00 & 7.00 & 7.00 & 0.22 & 3.90 & 3.90 & 3.90 \\
0.26 & 4.80 & 3.60 & 4.80 & 0.25 & 3.50 & 2.80 & 3.50 \\
0.28 & 4.40 & 2.60 & 4.15 & 0.30 & 3.00 & 1.50 & 2.80 \\
0.30 & 4.00 & 2.00 & 3.60 & 0.35 & 2.50 & 0.75 & 2.10 \\
0.35 & 3.30 & 1.30 & 2.80 & 0.40 & 2.10 & 0.45 & 1.60 \\
0.40 & 2.90 & 0.90 & 2.30 & 0.50 & 1.60 & 0.16 & 0.80 \\
0.50 & 2.30 & 0.50 & 1.60 & 0.55 & 1.38 & 0.08 & 0.58 \\
0.60 & 1.85 & 0.15 & 0.80 & 0.65 & 1.05 & 0.02 & 0.30 \\
0.65 & 1.65 & 0.00 & 0.00 & 0.70 & 0.93 & 0.01 & 0.22 \\
0.70 & 1.50 & & & 0.75 & 0.80 & 0.00 & 0.00 \\
0.80 & 1.20 & & & 0.90 & 0.55 & & \\
0.90 & 0.90 & & & 0.96 & 0.40 & & \\
0.99 & 0.40 & & & 0.99 & 0.19 & & \\
1.00 & 0.00 & & & 1.00 & 0.00 & & \\
\hline
\end{tabular}

Table A-9: PVT Data (Common for all examples):

\begin{tabular}{|llllll|}
\hline $\mathrm{p}$ & $\mathrm{z}$ & $\boldsymbol{\mu}_{\mathrm{g}}$ & $\mathrm{B}_{\mathrm{o}}$ & $\boldsymbol{\mu}_{\mathrm{o}}$ & $\mathrm{R}_{\mathrm{s}}$ \\
\hline 14.50 & 1.0000 & 0.0126 & 1.0279 & 0.9600 & 0.000 \\
37.10 & 0.9700 & 0.0129 & 1.0488 & 0.7250 & 7.930 \\
739.69 & 0.9440 & 0.0133 & 1.0644 & 0.6080 & 15.690 \\
1029.77 & 0.9260 & 0.0138 & 1.0766 & 0.5440 & 21.700 \\
1319.84 & 0.9100 & 0.0144 & 1.0887 & 0.4940 & 27.630 \\
1754.96 & 0.8930 & 0.0153 & 1.1065 & 0.4250 & 36.280 \\
2045.03 & 0.8860 & 0.0159 & 1.1176 & 0.4000 & 41.900 \\
2335.11 & 0.8840 & 0.0167 & 1.1293 & 0.3770 & 47.190 \\
2625.18 & 0.8850 & 0.0175 & 1.1402 & 0.3590 & 52.180 \\
2770.22 & 0.8870 & 0.0179 & 1.1435 & 0.3530 & 54.540 \\
7266.39 & 1.0000 & 0.0240 & 1.3000 & 0.1500 & 114.00 \\
\hline
\end{tabular}

Oil density: $890.6 \mathrm{~kg} / \mathrm{m}^{3}$; gas density: $0.741 \mathrm{~kg} / \mathrm{m}^{3}$ 
